Journal of Bioinformatics and Computational Biology

Vol. 14, No. 6 (2016) 1699001 (10 pages)

(c) World Scientific Publishing Europe Ltd.

DOI: $10.1142 /$ S0219720016990018

\title{
Author Index \\ Volume 14 (2016)
}

Abbasi, W. A. and Minhas, F. U. A. A., Issues in performance evaluation for host-pathogen protein interaction prediction

3 (2016) 1650011

Abidi, N., Franke, R., Findeisen, P. and Klawonn, F., Alignment of time-resolved data from high throughput experiments

6 (2016) 1650030

Akbal-Delibas, B., Farhoodi, R., Pomplun, M. and Haspel, N., Accurate refinement of docked protein complexes using evolutionary information and deep learning

Aksianov, E., see Zanegina, O.

Al Mahi, N. and Begum, M., A two-step integrated approach to detect differentially expressed genes in RNA-seq data

Alexeevski, A. V., see Zanegina, O.

Artemov, G. N., see Sharakhov, I. V.

Arzhanik, V., see Petrov, A.

Bashirov, R., see Mehraei, M.

3 (2016) 1642002

3 (2016) 1641007

6 (2016) 1650034

6 (2016) 1641007

6 (2016) 1630003

6 (2016) 1650019

6 (2016) 1650026

6 (2016) 1650034

Behaegel, J., Comet, J.-P., Bernot, G., Cornillon, E. and Delaunay, F., A hybrid model of cell cycle in mammals

Bernot, G., see Behaegel, J.

1 (2016) 1640001

1 (2016) 1640001

Bielecki, J. and Lipiec, E., Basis set dependence using DFT/B3LYP calculations to model the Raman spectrum of thymine

Biswal, D. K., see Konhar, R.

Biswas, A. K. and Gao, J. X., PR2S2Clust: Patched RNA-seq read segments' structure-oriented clustering

1 (2016) 1650002

1 (2016) 1660001

5 (2016) 1650027

Byun, Y. and Han, K., An efficient algorithm for planar drawing of RNA structures with pseudoknots of any type

3 (2016) 1650009

Cai, Z., see Li, J.

3 (2016) 1643001

Cao, C.-C. and Sun, X., Ehapp2: Estimate haplotype frequencies from pooled sequencing data with prior database information 
Cerqueda-García, D. and Falcón, L. I., Metabolic potential of microbial mats and microbialites: Autotrophic capabilities described by an in silico stoichiometric approach from shared genomic resources

4 (2016) 1650020

Chan, J. H., see Engchuan, W.

Chatterjee, P. and Pal, N. R., Discovery of synergistic genetic network: A minimum spanning tree-based approach

Chen, J., Pan, T., Pu, T., Huang, B., Huang, D. Y., Zhang, W., Gabos, S. and Jin, C., Predicting GHS toxicity using RTCA and discrete-time Fourier transform

4 (2016) 1650015

1 (2016) 1650003

Chen, J., see Jiang, L.

Chen, Y., see Zhao, Y.

1 (2016) 1650004

1 (2016) 1650005

1 (2016) 1650012

Chen, Y.-Y., see Pian, C.

Chen, Z., see Pian, C.

1 (2016) 1650006

1 (2016) 1650006

Chen, Z., see $\mathrm{Wu}, \mathrm{T}$.

1 (2016) 1650013

Choi, K., Smith, L. P., Medley, J. K. and Sauro, H. M., phraSED-ML: A paraphrased, human-readable adaptation of SED-ML

6 (2016) 1650035

Chowdhury, A., Rakshit, P. and Konar, A., Prediction of protein-protein interaction network using a multiobjective optimization approach

Christoffels, A. G., see Mapiye, D. S.

Comet, J.-P., see Behaegel, J.

Cong, W., see Li, J.

Cornillon, E., see Behaegel, J.

Dascalu, S. M., see Ferneyhough, G. B.

Debnath, M., see Konhar, R.

DeJesus, M. A. and Ioerger, T. R., Normalization of transposon-mutant library sequencing datasets to improve identification of conditionally essential genes

Delaunay, F., see Behaegel, J.

Deng, B., Kim, S., Li, H., Heath, E. and Zhang, X., Global peak alignment for comprehensive two-dimensional gas chromatography mass spectrometry using point matching algorithms

6 (2016) 1650032

6 (2016) 1650015

Embar, V., Handen, A. and Ganapathiraju, M. K., Is the average shortest path length of gene set a reflection of their biological relatedness?

Engchuan, W., Meechai, A., Tongsima, S., Doungpan, N. and Chan, J. H., Gene-set activity toolbox (GAT): A platform for microarray-based cancer diagnosis using an integrative gene-set analysis approach

3 (2016) 1650008

3 (2016) 1650022

3 (2016) 1640001

3 (2016) 1643001

3 (2016) 1640001

3 (2016) 1642003

3 (2016) 1660001

3 (2016) 1642004

3 (2016) 1640001

6 (2016) 1660002

4 (2016) 1650015 
Ershova, A., Rusinov, I., Vasiliev, M., Spirin, S. and Karyagina, A., Restriction-Modification systems interplay causes avoidance of GATC site in prokaryotic genomes

Eulenstein, O., see Moon, J.

Falcón, L. I., see Cerqueda-García, D.

Farhoodi, R., see Akbal-Delibas, B.

Feng, W., see Li, J.

Ferneyhough, G. B., Thibealut, C. M., Dascalu, S. M. and Harris Jr., F. C., ModFossa: A library for modeling ion channels using Python

Findeisen, P., see Abidi, N.

Franke, R., see Abidi, N.

Fudenberg, G., see Khrameeva, E. E.

Gabos, S., see Chen, J.

Galzitskaya, O. V., see Shiliaev, N. G.

Gamieldien, J., see Mapiye, D. S.

Ganapathiraju, M. K., see Embar, V.

Gao, J. X., see Biswas, A. K.

Gelfand, M. S., see Kalinina, A. S.

Gelfand, M. S., see Khrameeva, E. E.

Geller, J., see Ochs, C.

Gerstgrasser, M., Nicholls, S., Stout, M., Smart, K., Powell, C., Kypraios, T. and Stekel, D., A Bayesian approach to analyzing phenotype microarray data enables estimation of microbial growth parameters

Gim, J., Won, S. and Park, T., Conditional estimation of local pooled dispersion parameter in small-sample RNA-Seq data improves differential expression test

Goh, W. W. B. and Wong, L., Evaluating feature-selection stability in next-generation proteomics

Goh, W. W. B. and Wong, L., Spectra-first feature analysis in clinical proteomics - A case study in renal cancer

Göhlmann, H. W. H., see Perualila-Tan, N. J.

Gong, F., see $\mathrm{Wu}, \mathrm{T}$.

González, J., Muñoz, A. and Martos, G., Asymmetric latent semantic indexing for gene expression experiments visualization

Gu, L., Xue, L., Song, Q., Wang, F., He, H. and Zhang, Z., Classification of the fragrant styles and evaluation of the aromatic quality of flue-cured tobacco leaves by machine-learning methods

Halper, M., see Ochs, C.
2 (2016) 1641003

2 (2016) 1642005

2 (2016) 1650020

2 (2016) 1642002

2 (2016) 1643001

3 (2016) 1642003

3 (2016) 1650030

3 (2016) 1650030

3 (2016) 1641002

3 (2016) 1650004

3 (2016) 1641004

3 (2016) 1650022

3 (2016) 1660002

3 (2016) 1650027

3 (2016) 1641001

3 (2016) 1641002

3 (2016) 1642001

3 (2016) 1650007

5 (2016) 1644006

5 (2016) 1650029

5 (2016) 1644004

5 (2016) 1650018

5 (2016) 1650013

4 (2016) 1650023

6 (2016) 1650033

6 (2016) 1642001 
Han, J., see Wang, X.

6 (2016) 1650014

Han, K., see Byun, Y.

6 (2016) 1650009

Han, W., see Park, J.

6 (2016) 1644002

Handen, A., see Embar, V.

6 (2016) 1660002

Hao, F., see Yu, S.

Hao, M., see Wang, X.

6 (2016) 1640002

Harris Jr., F. C., see Ferneyhough, G. B.

6 (2016) 1650014

6 (2016) 1642003

Haspel, N., see Akbal-Delibas, B.

6 (2016) 1642002

He, D., Huang, L., Xu, Y., Pan, X. and Liu, L., Computational analysis and enzyme assay of inhibitor response to disease single nucleotide polymorphisms (SNPs) in lipoprotein lipase

He, H., see $\mathrm{Gu}$, L.

He, Y., see Jiang, L.

5 (2016) 1650028

5 (2016) 1650033

5 (2016) 1650005

5 (2016) 1650032

Heath, E., see Deng, B.

5 (2016) 1641005

$\mathrm{Hu}, \mathrm{R}$. and Sun, X., lncRNATargets: A platform for lncRNA target prediction based on nucleic acid thermodynamics

Huang, B., see Chen, J.

Huang, D. Y., see Chen, J.

Huang, L., see He, D.

Huh, I., see Oh, S.

Hur, B., see Park, J.

Ikram, A. A., see Sardaraz, M.

Ioerger, T. R., see DeJesus, M. A.

Jiang, L., Chen, J., He, Y., Zhang, Y. and Li, G., A method to predict different mechanisms for blood-brain barrier permeability of CNS activity compounds in Chinese herbs using support vector machine

Jiang, M., see Zhao, Y.

4 (2016) 1650016

4 (2016) 1650004

4 (2016) 1650004

4 (2016) 1650028

4 (2016) 1644005

4 (2016) 1644002

4 (2016) 1630002

4 (2016) 1642004

Jin, C., see Chen, J.

Kalinina, A. S., Suvorikova, A. L., Spokoiny, V. G. and

Gelfand, M. S., Detection of homologous recombination in closely related strains

Karyagina, A., see Ershova, A.

2 (2016) 1641001

2 (2016) 1641003

2 (2016) 1641007

Karyagina, A., see Zanegina, O.

1 (2016) 1650005

1 (2016) 1650012

1 (2016) 1650004

Kasim, A., see Perualila-Tan, N. J.

2 (2016) 1650018

2 (2016) 1650010

Khrameeva, E. E., Fudenberg, G., Gelfand, M. S. and Mirny,

L. A., History of chromosome rearrangements reflects the spatial organization of yeast chromosomes

2 (2016) 1641002

Kim, K., see Park, J.

2 (2016) 1644002 
Kim, M.-S., see Park, J.

2 (2016) 1644002

Kim, S., see Deng, B.

2 (2016) 1650032

Kim, S., see Park, J.

2 (2016) 1644002

Klawonn, F., see Abidi, N.

2 (2016) 1650030

Kodama, K. and Saigo, H., KDSNP: A kernel-based

approach to detecting high-order SNP interactions

5 (2016) 1644003

5 (2016) 1650019

Koliasnikov, O., see Petrov, A.

5 (2016) 1650008

5 (2016) 1641006

Kondrakhin, Y. V., see Volkova, O. A.

Konhar, R., Debnath, M., Marbaniang, J. V., Biswal, D. K. and Tandon, P., Age estimation for the genus Cymbidium (Orchidaceae: Epidendroideae) with implementation of fossil data calibration using molecular markers (ITS2 \& matK) and phylogeographic inference from ancestral area reconstruction

6 (2016) 1660001

Kypraios, T., see Gerstgrasser, M.

6 (2016) 1650007

Lee, S. Y., see Oh, S.

6 (2016) 1644005

Leong, H. W., see Lu, B.

6 (2016) 1640003

6 (2016) 1640002

6 (2016) 1641009

Levitsky, V. G., see Zemlyanskaya, E. V.

6 (2016) 1650005

Li, G., see Jiang, L.

6 (2016) 1650032

Li, J., see Shen, F.

6 (2016) 1644001

Li, J., Xu, C., Wang, L., Liang, H., Feng, W., Cai, Z., Wang, Y., Cong, W. and Liu, Y., PSRna: Prediction of small RNA secondary structures based on reverse complementary folding method

4 (2016) 1643001

4 (2016) 1650014

Li, K., see Wang, X.

4 (2016) 1650006

Li, Q., see Pian, C.

4 (2016) 1643001

Liang, H., see Li, J.

4 (2016) 1644002

Lin, H. T., see Moon, J.

4 (2016) 1642005

4 (2016) 1650002

Lipiec, E., see Bielecki, J.

4 (2016) 1650028

Liu, S., see Yang, B.

4 (2016) 1650021

4 (2016) 1643001

Liu, Y., see Li, J.

4 (2016) 1642001

Lu, B. and Leong, H. W., GI-SVM: A sensitive method for predicting genomic islands based on unannotated sequence of a single genome

1 (2016) 1640003

Makarov, G., see Petrov, A.

1 (2016) 1650019 
Mandal, S., Khan, A., Saha, G. and Pal, R. K., Reverse engineering of gene regulatory networks based on S-systems and Bat algorithm

3 (2016) 1650010

Mapiye, D. S., Christoffels, A. G. and Gamieldien, J., Identification of phenotype-relevant differentially expressed genes in breast cancer demonstrates enhanced quantile discretization protocol's utility in multi-platform microarray data integration

Marbaniang, J. V., see Konhar, R.

Martos, G., see González, J.

Medley, J. K., see Choi, K.

Meechai, A., see Engchuan, W.

Mehraei, M., Bashirov, R. and Tüzmen, Ş., Target-based drug discovery for $\beta$-globin disorders: Drug target prediction using quantitative modeling with hybrid functional Petri nets

Minhas, F. U. A. A., see Abbasi, W. A.

Mirny, L. A., see Khrameeva, E. E.

Mironova, V. V., see Zemlyanskaya, E. V.

Moerbeke, M. V., see Perualila-Tan, N. J.

Moon, J., Lin, H. T. and Eulenstein, O., Consensus properties and their large-scale applications for the gene duplication problem

Muñoz, A., see González, J.

Myasnikova, E. M., see Spirov, A. V.

Nicholls, S., see Gerstgrasser, M.

Ochs, C., Perl, Y., Halper, M., Geller, J. and Lomax, J., Quality assurance of the gene ontology using abstraction networks

Oh, S., Huh, I., Lee, S. Y. and Park, T., Analysis of multiple 5 (2016) 1650022 5 (2016) 1660001

5 (2016) 1650023

5 (2016) 1650035

5 (2016) 1650015

5 (2016) 1650026

5 (2016) 1650011

5 (2016) 1641002

5 (2016) 1641009

5 (2016) 1650018 related phenotypes in genome-wide association studies

Omelyanchuk, N. A., see Zemlyanskaya, E. V.

Pal, N. R., see Chatterjee, P.

Pal, R. K., see Mandal, S.

Pan, T., see Chen, J.

3 (2016) 1642005

3 (2016) 1650023

3 (2016) 1641005

3 (2016) 1650007

Pan, X., see He, D.

Park, J., Hur, B., Rhee, S., Lim, S., Kim, M.-S., Kim, K., Han, W. and Kim, S., Information theoretic sub-network mining characterizes breast cancer subtypes in terms of cancer core mechanisms 
Pavel, A. B. and Vasile, C. I., Identifying cancer type specific oncogenes and tumor suppressors using limited size data

Perl, Y., see Ochs, C.

6 (2016) 1650031

6 (2016) 1642001

Perualila-Tan, N. J., Shkedy, Z., Talloen, W., Göhlmann, H. W. H., Quantitative Structure Transcription Assay Relationships (QSTAR) Consortium, Moerbeke, M. V. and Kasim, A., Weighted similarity-based clustering of chemical structures and bioactivity data in early drug discovery

4 (2016) 1650018

Petrov, A., Arzhanik, V., Makarov, G. and Koliasnikov, O., A novel Arg H52/Tyr H33 conservative motif in antibodies: A correlation between sequence of antibodies and antigen binding

Pian, C., Zhang, J., Chen, Y.-Y., Chen, Z., Li, Q., Li, Q. and 4 (2016) 1650019 Zhang, L.-Y., OP-Triplet-ELM: Identification of real and pseudo microRNA precursors using extreme learning machine with optimal features

1 (2016) 1650006

Pomplun, M., see Akbal-Delibas, B.

Popova, N., see Suplatov, D.

Powell, C., see Gerstgrasser, M.

Precharattana, M., Stochastic modeling for dynamics of HIV-1 infection using cellular automata: A review

$\mathrm{Pu}$, T., see Chen, J.

Quantitative Structure Transcription Assay Relationships (QSTAR) Consortium, see Perualila-Tan, N. J.

Rakshit, P., see Chowdhury, A.

Rhee, S., see Park, J.

Rusinov, I., see Ershova, A.

1 (2016) 1642002

1 (2016) 1641008

1 (2016) 1650007

1 (2016) 1630001

1 (2016) 1650004

1 (2016) 1650018

1 (2016) 1650008

1 (2016) 1644002

1 (2016) 1641003

Saha, G., see Mandal, S.

Saigo, H., see Kodama, K.

1 (2016) 1650010

1 (2016) 1644003

Sardaraz, M., Tahir, M. and Ikram, A. A., Advances in high throughput DNA sequence data compression

3 (2016) 1630002

3 (2016) 1650035

3 (2016) 1641004

Selivanova, O. M., see Shiliaev, N. G.

Sharakhov, I. V., Artemov, G. N. and Sharakhova, M. V., Chromosome evolution in malaria mosquitoes inferred from physically mapped genome assemblies

2 (2016) 1630003

2 (2016) 1630003

Sharakhova, M. V., see Sharakhov, I. V.

2 (2016) 1641006

Shen, F., Li, J., Zhu, Y. and Wang, Z., Systematic investigation of metabolic reprogramming in different cancers based on tissue-specific metabolic models 
Shiliaev, N. G., Selivanova, O. M. and Galzitskaya, O. V., Search for conserved amino acid residues of the $\alpha$-crystallin proteins of vertebrates

Shkedy, Z., see Perualila-Tan, N. J.

Smart, K., see Gerstgrasser, M.

Smith, L. P., see Choi, K.

Song, Q., see $\mathrm{Gu}$, L.

Song, T., see $\mathrm{Wu}, \mathrm{T}$.

Spirin, S., see Ershova, A.

Spirin, S., see Zanegina, O.

Spirov, A. V., Myasnikova, E. M. and Holloway, D. M., Sequential construction of a model for modular gene expression control, applied to spatial patterning of the Drosophila gene hunchback

Spokoiny, V. G., see Kalinina, A. S.

Stekel, D., see Gerstgrasser, M.

Stout, M., see Gerstgrasser, M.

Sun, T., see $\mathrm{Wu}, \mathrm{W}$.

Sun, W., see Wu, W.

Sun, X., see Cao, C.-C.

Sun, X., see $\mathrm{Hu}, \mathrm{R}$.

Suplatov, D., Popova, N., Zhumatiy, S., Voevodin, V. and Švedas, V., Parallel workflow manager for non-parallel bioinformatic applications to solve large-scale biological problems on a supercomputer

Suvorikova, A. L., see Kalinina, A. S.

Švedas, V., see Suplatov, D.

Tahir, M., see Sardaraz, M.

Talloen, W., see Perualila-Tan, N. J.

Tandon, P., see Konhar, R.

Thibealut, C. M., see Ferneyhough, G. B.

Tongsima, S., see Engchuan, W.

Tüzmen, Ş., see Mehraei, M.

Valeev, T. F., see Volkova, O. A.

Vasile, C. I., see Pavel, A. B.

Vasiliev, M., see Ershova, A.

Voevodin, V., see Suplatov, D.

Volkova, O. A., Kondrakhin, Y. V., Yevshin, I. S., Valeev, T. F. and Sharipov, R. N., Assessment of translational importance of mammalian mRNA sequence features based on Ribo-Seq and mRNA-Seq data

Wang, F., see $\mathrm{Gu}$, L.
2 (2016) 1641004

2 (2016) 1650018

2 (2016) 1650007

2 (2016) 1650035

2 (2016) 1650033

2 (2016) 1650013

2 (2016) 1641003

2 (2016) 1641007

2 (2016) 1641005

2 (2016) 1641001

2 (2016) 1650007

2 (2016) 1650007

2 (2016) 1650001

2 (2016) 1650001

2 (2016) 1650017

2 (2016) 1650016

2 (2016) 1641008

2 (2016) 1641001

2 (2016) 1641008

2 (2016) 1630002

2 (2016) 1650018

2 (2016) 1660001

2 (2016) 1642003

2 (2016) 1650015

2 (2016) 1650026

2 (2016) 1641006

2 (2016) 1650031

2 (2016) 1641003

2 (2016) 1641008

2 (2016) 1641006

2 (2016) 1650033 
Wang, G., see Wang, X.

2 (2016) 1650014

Wang, L., see Li, J.

2 (2016) 1643001

Wang, X., Han, J., Li, K., Wang, G. and Hao, M., Multi-layer composite mechanical modeling for the inhomogeneous biofilm mechanical behavior

4 (2016) 1650014

Wang, X., see $\mathrm{Wu}, \mathrm{T}$.

Wang, Y., see Li, J.

4 (2016) 1650013

4 (2016) 1643001

Wang, Z., see Shen, F.

Wiebe, D. S., see Zemlyanskaya, E. V.

4 (2016) 1644001

4 (2016) 1641009

Wong, L., see Goh, W. W. B.

4 (2016) 1644004

Wong, L., see Goh, W. W. B.

4 (2016) 1650029

Won, S., see Gim, J.

Wu, T., Wang, X., Zhang, Z., Gong, F., Song, T., Chen, Z., 4 (2016) 1644006 Zhang, P. and Zhao, Y., NES-REBS: A novel nuclear export signal prediction method using regular expressions and biochemical properties

Wu, W., Sun, W. and Sun, T., Modeling the heterogeneity of p53 dynamics in DNA damage response

3 (2016) 1650013

1 (2016) 1650001

$\mathrm{Xu}, \mathrm{C}$., see $\mathrm{Li}, \mathrm{J}$.

$\mathrm{Xu}, \mathrm{Y}$., see He, D.

1 (2016) 1643001

1 (2016) 1650028

Xue, L., see $\mathrm{Gu}$, L.

1 (2016) 1650033

Yang, B., Liu, S. and Zhang, W., Reverse engineering of gene regulatory network using restricted gene expression programming

Yevshin, I. S., see Volkova, O. A.

$\mathrm{Yu}$, S., Hao, F. and Leong, H. W., An $\mathrm{O}\left(n^{3}\right)$ algorithm for sorting signed genomes by reversals, transpositions, transreversals and block-interchanges

Zanegina, O., Aksianov, E., Alexeevski, A. V., Karyagina, A. and Spirin, S., Conserved features of complexes of TATA-box binding proteins with DNA

Zemlyanskaya, E. V., Wiebe, D. S., Omelyanchuk, N. A., Levitsky, V. G. and Mironova, V. V., Meta-analysis of transcriptome data identified TGTCNN motif variants associated with the response to plant hormone auxin in Arabidopsis thaliana $L$.

5 (2016) 1650021

5 (2016) 1641006

1 (2016) 1640002

Zhang, J., see Pian, C.

2 (2016) 1641009

2 (2016) 1650006

Zhang, L.-Y., see Pian, C.

2 (2016) 1650006

Zhang, P., see $\mathrm{Wu}, \mathrm{T}$.

2 (2016) 1650013

Zhang, W., see Chen, J.

2 (2016) 1650004

Zhang, W., see Yang, B.

2 (2016) 1650021

Zhang, X., see Deng, B.

2 (2016) 1650032 
Zhang, Y., see Jiang, L.

2 (2016) 1650005

Zhang, Z., see Gu, L.

2 (2016) 1650033

Zhang, Z., see $\mathrm{Wu}, \mathrm{T}$.

2 (2016) 1650013

Zhao, Y., Jiang, M. and Chen, Y., Inferring gene regulatory

4 (2016) 1650012 networks using a time-delayed mass action model

4 (2016) 1650013

Zhao, Y., see $\mathrm{Wu}, \mathrm{T}$.

4 (2016) 1644001

Zhu, Y., see Shen, F.

4 (2016) 1641008

Zhumatiy, S., see Suplatov, D. 